\section{Total occlusion of the esophagus following placement of an over-the-scope clip: an unusual complication, and how to solve it}

The over-the-scope clip (OTSC; Ovesco Endoscopy AG, Tübingen, Germany) has recently proven to be of great benefit for the closure of gastrointestinal defects, especially fistula following bariatric surgery [1]. Until recently, there were no good methods of removing the OTSC after unintended placement, but a newly developed dedicated cutter has proven to be efficient [2].

A 42-year-old man who underwent sleeve gastrectomy developed a 13-mm chronic fistula at the angle of His, and an abscess into the left mediastinum. The fistula was initially unsuccessfully treated by placement of a fully covered stent. Closure with an OTSC-gc was then attempted after de-epithelialization of the edges of the fistula with argon plasma coagulation (APC2; Erbe Elektromedizin, Tübingen, Germany). After the edges of the fistula had been anchored with the Ovesco Twin grasper and pulled, suction was applied before the release of the clip. The result was total closure of the esophageal lumen, as a result of too much mucosal tissue being sucked into the cap. The total occlusion was confirmed by contrast examination ( Fig. 1). Attempts to cut the clip with APC at the maximum setting were unsuccessful and resulted in severe thermal damage to the surrounding tissue ( $\bullet$ Video 1 ).

The patient fasted for 24 hours while a newly developed and commercialized DC ClipCutter (Ovesco Endoscopy AG) was acquired. By using this cutting device, it was possible to cut the OTSC into four sections ( Fig.2) and remove it ( $\bullet$ Fig.3, - Video 1).

Following removal of the clip, the surrounding tissue was swollen and inflamed, rendering the area temporarily inaccessible for further evaluation and intervention. A covered stent was placed to bypass the fistula for a few weeks, prior to a new attempt to close the fistula.

To our knowledge, this is the first description of an iatrogenic total closure of the esophagus with an OTSC. Such emergencies require a dedicated cutting device. Another lesson learned was to avoid suction when using the OTSC in the esophagus.

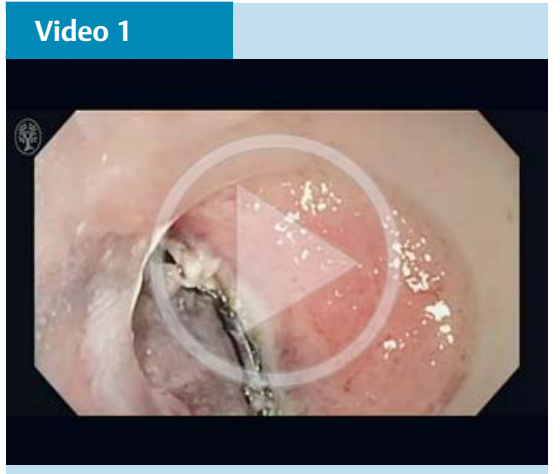

Total occlusion of the esophagus following placement of an over-the-scope clip. Attempts to cut the clip using argon plasma coagulation failed, and resulted in severe thermal injury to the surrounding tissue. A DC ClipCutter (Ovesco Endoscopy AG, Tübingen, Germany) was finally acquired to cut through the clip and facilitate removal. A covered stent was placed to bypass the fistula for a few weeks, prior to a new attempt to close the fistula.

Endoscopy_UCTN_Code_CPL_1AH_2AG

Competing interests: None

\section{Khanh Do-Cong Pham ${ }^{1,2}$, Georg G. Dimcevski ${ }^{1,2}$}

${ }^{1}$ Department of Medicine, Haukeland University Hospital, Bergen, Bergen, Norway 2 Department of Clinical Medicine, K1, University of Bergen, Bergen, Norway

\section{References}

1 Mercky P, Gonzalez J-M, Aimore Bonin E et al. Usefulness of over-the-scope clipping system for closing digestive fistulas. Dig Endosc 2015; $1: 18-24$

2 Schmidt A, Riecken B, Damm $M$ et al. Endoscopic removal of over-the-scope clips using a novel cutting device: a retrospective case series. Endoscopy 2014; 9: 762 - 766

\section{Bibliography}

DOI http://dx.doi.org/

10.1055/s-0042-105209

Endoscopy 2016; 48: E128

(c) Georg Thieme Verlag KG

Stuttgart · New York

ISSN 0013-726X

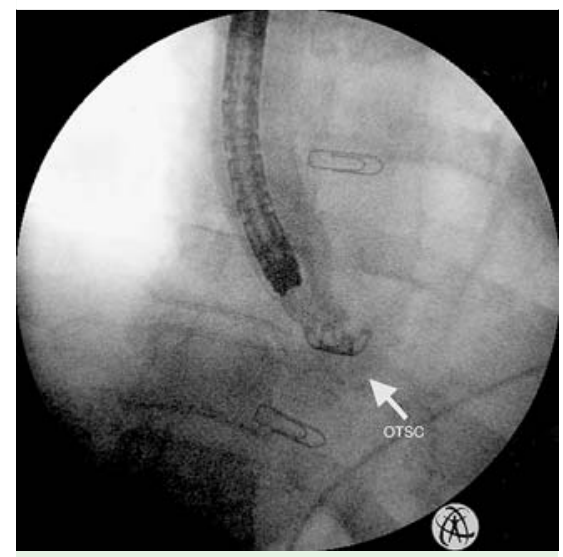

Fig. 1 There was no passage of contrast across the over-the-scope clip (arrow).

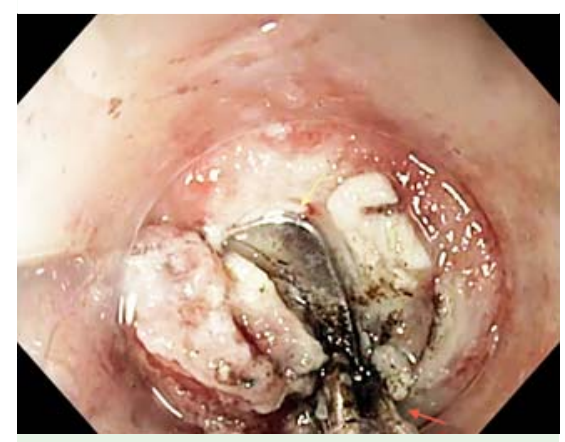

Fig. 2 Cutting the over-the-scope clip (yellow) with the cutting device (red arrow). The surrounding tissue showed severe thermal damage caused by previous attempts to cut the clip using argon plasma coagulation.

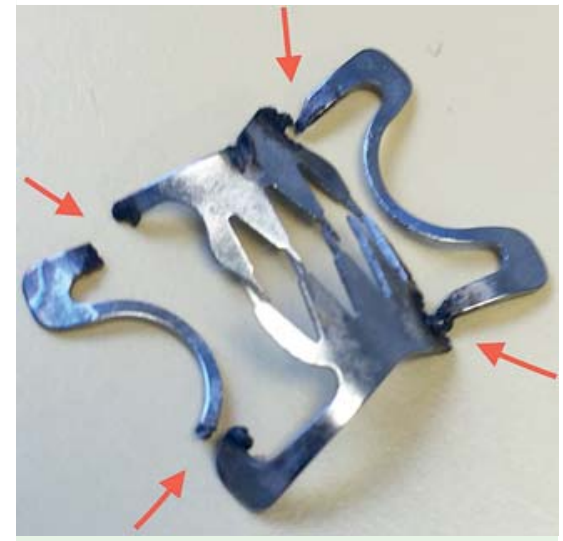

Fig. 3 The over-the-scope clip after cutting (arrows) and removal.

\section{Corresponding author} Khanh Do-Cong Pham, MD

Section of Gastroenterology

Department of Medicine

Haukeland University Hospital

Jonas Lies vei 65

5051 Bergen

Norway

Fax: +47-55955000

phamkdc@gmail.com 